\title{
Risoluzione dell'ipercortisolismo da secrezione ectopica di ACTH in un paziente con carcinoma midollare della tiroide metastatico con l'impiego di sorafenib
}

\author{
Emanuela Arvat
}

Pubblicato online: 28 gennaio 2015

(C) Springer International Publishing AG 2015

Commento a:

Complete resolution of hypercortisolism with sorafenib in a patient with advanced medullary thyroid carcinoma and ectopic ACTH syndrome.

R. Barroso-Sousa, A. Lerario, J. Evangelista, C. Papadia, D.M. Lourenco, C.S. Lin, M.A. Kulcsar, M.C. Fragoso, A.O. Hoff.

Thyroid (2014) 6:1062-1066

La sindrome da ACTH ectopico rappresenta una rara manifestazione di secrezione ormonale ectopica, tipica dei tumori neuroendocrini, in grado di influenzare negativamente la prognosi della malattia principale. Essa viene generalmente trattata con i farmaci comunemente utilizzati per il controllo dell'ipercortisolismo, in particolare farmaci inibitori della steroidogenesi o mitotane; tuttavia, il controllo di malattia in questa condizione clinica risulta spesso transitorio o inadeguato.

Analogamente a quanto accade per altri tumori maligni della tiroide e per numerosi tumori neuroendocrini, il tumore midollare della tiroide, in fase avanzata e metastatica, ha attualmente come indicazione terapeutica la terapia medica a bersaglio molecolare, in particolare con farmaci inibitori di tirosino-chinasi, quali il vandetanib e il cabozantinib. Tuttavia, altri farmaci della stessa famiglia, quali il sorafenib, sono stati recentemente utilizzati nel trattamenti di questi tipi di tumore.

Lo studio in oggetto ha riportato l'efficacia clinica e biochimica di un trattamento prolungato con sorafenib $(400 \mathrm{mg}$ $\times 2 /$ die, durata circa 1 anno) nel controllare in modo com- pleto la condizione di ipercortisolismo da secrezione ectopica di ACTH in un paziente con carcinoma midollare della tiroide in fase metastatica. Tale effetto sulla secrezione ormonale si è manifestato in associazione a una condizione di stabilizzazione di malattia, della durata di circa 1 anno, epoca in cui si è manifestata una progressione della patologia oncologica e una perdita del controllo sulla secrezione di cortisolo.

Durante la terapia, le condizioni cliniche del paziente, in particolare quelle relative allo stato di ipercortisolismo, sono nettamente migliorate con un miglioramento della qualità di vita.

I dati dello studio indicano quindi, analogamente a quanto già osservato per altri farmaci inibitori di tirosino-chinasi, la capacità di queste molecole di ridurre l'ipersecrezione di ACTH e cortisolo in corso di sindrome ormonale ectopica. Numerosi meccanismi sono stati ipotizzati per spiegare tale effetto (inibizione diretta della sintesi di ACTH, inibizione della steroidogenesi, induzione di ischemia surrenalica). Dal punto di vista clinico, l'utilizzo di queste molecole potrebbe essere tenuto in considerazione in presenza di sindrome da ACTH ectopico in tumori in fase avanzata in cui vi sia l'indicazione al loro utilizzo, allo scopo di controllare contemporaneamente sia la progressione di malattia che l'ipersecrezione ormonale. Inoltre, la possibile induzione di iposurrenalismo potrebbe spiegare la presenza di alcuni effetti collaterali frequentemente indotti dalla terapia con questi farmaci; pertanto, la funzione corticosurrenalica dovrebbe essere valutata in questi pazienti.
E. Arvat $(\otimes)$

Divisione di Endocrinologia Oncologica, Dipartimento di Scienze

Mediche, Università di Torino, Torino, Italia

e-mail: emanuela.arvat@unito.it 